\title{
A transgender refugee woman experiencing posttraumatic stress disorder symptoms and homelessness
}

\author{
Alex Abramovich MA PhD, June Sing Hong Lam MD, Muna Chowdhury MD
}

Cite as: CMAJ 2020 January 6;192:E9-11. doi: 10.1503/cmaj.190974

A 23-year-old transgender woman was referred to a community health centre and presented with a long history of gender dysphoria and desire to medically transition. She disclosed an extensive history of trauma and that she had experienced recurrent nightmares, insomnia, hypervigilance, depressive symptoms, dissociative symptoms and social isolation. The patient was a recent refugee claimant, born and raised in Saudi Arabia. She had minimal English proficiency and was residing in an emergency shelter. She had difficulty accessing supportive health care and navigating the complex refugee system. She was eventually connected with the clinic and through interpretation services was able to tell her story.

The patient had identified as transgender at an early age. On discovering that she was transgender, her father attempted to murder her. She managed to escape and was subsequently apprehended by law enforcement officers, and reported being sexually assaulted by them. Once released, the patient continued to be harassed on a daily basis by people on the street and in her neighbourhood, from name-calling to being beaten to having her long hair shaved off by a mob. With her friends' support, she immigrated to Toronto, where she struggled to navigate the complex social service and health care systems because of language barriers, cultural differences, homelessness and poverty.

Over multiple visits to her physician at the community health centre, the patient disclosed more about herself, including that being born with male genitalia had caused her substantial distress since childhood and that puberty was especially difficult because of increased facial and body hair growth, deepening of her voice and penile erections. She shared her desire to live as a woman and described expressing her gender identity through feminine clothing, makeup and jewellery. She disclosed that she had experienced passive suicidal ideation and hopelessness for many years. Gender dysphoria was diagnosed.

The patient underwent a physical examination and baseline screening blood tests, with normal findings. Because she requested and met readiness criteria for initiating hormone therapy, her physician prescribed spironolactone $25 \mathrm{mg}$, an androgen blocker. Four weeks later, the patient reported some clinical benefit, including reduced facial hair, and no adverse effects. Spironolactone was increased to $50 \mathrm{mg}$, and estradiol was started at $1 \mathrm{mg}$.

\section{KEY POINTS}

- Transgender individuals experience high rates of violence, discrimination and homelessness.

- Psychiatric disorders should be managed before or concurrent with medical transition for gender dysphoria.

- Posttraumatic stress disorder (PTSD) can be a psychiatric comorbidity for transgender individuals who frequently experience recurrent trauma; intersectional oppression and social determinants of health need to be addressed for adequate treatment of PTSD.

- Addressing homelessness and other social determinants of health requires long-term engagement with interdisciplinary team support.

- Access to medical transition has been associated with significantly reduced suicidality.

The patient returned for regular clinical follow-up and blood tests. Over a period of 6 months, spironolactone was increased to $100 \mathrm{mg}$ twice daily and estradiol was increased to $3 \mathrm{mg}$ twice daily, on the basis of a hormone therapy guideline from the Sherbourne Health Centre,$^{1}$ the patient's hormone levels and her desired effects. The patient experienced facial feminization, softening of skin, some breast development, decreased spontaneous erections, and reduction of facial and body hair; however, she continued to experience dysphoria about her genitals. After discussing transition-related surgical options (TRS, also known as genderaffirming surgery), she was referred for vaginoplasty.

While she underwent treatment for gender dysphoria, she was concurrently referred for a psychiatric evaluation, given her history of severe trauma and symptoms of mental illness. Posttraumatic stress disorder (PTSD) was diagnosed. In addition to weekly psychotherapy, she was prescribed mirtazapine $30 \mathrm{mg}$, taken at bedtime, for PTSD and insomnia, and prazosin $1 \mathrm{mg}$, taken at bedtime, for nightmares. The medication doses were gradually increased until she was stable at a dose of mirtazapine $60 \mathrm{mg}$ and prazosin $6 \mathrm{mg}$. She reported improved sleep, reduced PTSD symptoms and suicidal thoughts, and feeling more hopeful about the future.

The care team integrated a social determinants of healthbased approach into her treatment plan. She was referred to a 
housing worker to assist her in securing long-term stable housing. Accessing housing was extremely challenging because of a lack of affordable housing in Toronto. She temporarily stayed in emergency shelters but had to leave because of continuous transphobic discrimination and harassment, which threatened her safety. The patient became a sex worker and exchanged sex for food and shelter; however, she experienced ongoing violence. She is currently on a wait list for subsidized housing.

She was referred to a transgender community-based drop-in program to encourage connection to community, but the language and cultural barriers prevented her from feeling that she belonged. This led to her case manager connecting her with an English-as-a-second-language school to improve proficiency in English. A lawyer helped the patient gain permanent resident status in Canada, and her care team completed forms to change her sex marker and name on her government-issued identification.

The patient is continuing to focus on addressing both the PTSD and gender dysphoria. Her symptoms and social situation led her to sometimes miss appointments and medication doses. Though her condition is improved, she continues to experience nightmares and difficulty trusting others. She has had to postpone pursuing vaginoplasty because her precarious housing situation currently precludes her from having a good aftercare plan. A highly regimented aftercare plan is required after vaginoplasty, which involves vaginal douching and dilation as often as 4 times per day, gradually decreasing in frequency over time. As with any surgery, there is a risk of wound infection, particularly after the vaginoplasty. Patients are at risk for vaginitis and urinary tract infections, especially if hygiene is not maintained. Lack of stable housing adds an extra challenge to ensure adherence to the postvaginoplasty protocol.

\section{Discussion}

\section{Epidemiology}

"Transgender" is a term used to describe individuals whose gender identity does not align with their sex assigned at birth. Population studies estimate that $0.5 \%$ to $1.3 \%$ of adults (about 25 million people worldwide) identify as transgender, with transgender people in every country. ${ }^{2}$ It is well documented that transgender individuals face multiple barriers accessing health care, housing, employment and education, as a result of stigma, discrimination and structural violence. ${ }^{3-5}$ They experience negative physical and mental health outcomes and high rates of disease burden, including a high prevalence of mental health issues, suicide, depression, anxiety, and substance use, as well as HIV and other sexually transmitted infections. ${ }^{3-5}$ An estimated $44 \%$ of transgender people have experienced violence or victimization, suggesting an increased likelihood of PTSD; however, few studies have examined this. ${ }^{4}$

Lesbian, gay, bisexual, transgender, queer, questioning, and 2-spirit (LGBTQ2S) young people are overrepresented among youth experiencing homelessness. The Canadian definition of youth homelessness defines youth as being between the ages of 13 and 24 years. ${ }^{6}$ A recent national youth homelessness survey of 1103 respondents reported that about $30 \%$ of youth experiencing homelessness in Canada identify as LGBTQ2S. ${ }^{6}$ Accurate prevalence rates are dependent on the collection of inclusive data (e.g., ensuring all surveys and questionnaires include questions on sexual orientation and gender identity).

In the Trans PULSE Project, researchers investigated the health and well-being of transgender people living in Ontario, Canada, through surveys administered to 433 transgender people older than 16 years, with a wide range of intersectional identities. ${ }^{3}$ Numerous important findings were reported, including that $77 \%$ of transgender individuals in Ontario had seriously considered suicide and $43 \%$ had attempted suicide at some point in their lives, and that individuals experiencing high levels of transphobia were at greater risk of suicidality. ${ }^{7}$ A correlation was found between medical transition status and suicidality; for example, transgender individuals who were planning to medically transition, but who had not yet started, considered and attempted suicide more frequently than those who had already completed TRS or were not planning to medically transition. ${ }^{7}$ These individuals were also about 6 times more likely to experience depressive symptomatology than their counterparts who had already started their medical transition. ${ }^{8}$

\section{Interventions}

Intersectional oppression and social determinants of health should be addressed for adequate treatment. Transgender individuals are frequently subjected to discrimination, rejection and violence in housing programs, resulting in them avoiding services altogether. ${ }^{9}$ Institutional rules and policies that fail to recognize transgender identities perpetuate erasure (institutional rules that fail to recognize transgender identities and deliver an unspoken message that to be transgender is unacceptable), discrimination and stigma, also making it difficult for transgender youth to access housing and support services. ${ }^{9}$ Health care providers can support transgender youth experiencing homelessness by referring them to transgender-inclusive services and housing programs. Case managers and housing workers are often key members of the support team and can help transgender clients navigate services and advocate for inclusive resources.

Among transgender individuals who desire medical transition, access to hormones and TRS is a key protective factor against depression and suicide. ${ }^{7}$ Transgender people who have undergone TRS have a significantly reduced rate of suicide attempts. ${ }^{10}$ Physicians can help improve mental health and suicidality by facilitating access to desired medical transition by prescribing hormones and referring to specialists for TRS. ${ }^{7} \mathrm{Hav}$ ing a concurrent psychiatric condition should not preclude access to medical transition but needs to be optimally managed. ${ }^{1}$ It is important to treat psychiatric conditions concurrently with evidence-based treatments for medical transition.

Providers can refer transgender youth to community-based organizations, where they can connect with community members for support, because high levels of social support reduce the likelihood of a suicide attempt. ${ }^{7}$ Having 1 or more governmentissued identification document (e.g., passport, driver's licence) concordant with a transgender person's lived gender has also been associated with almost half the risk of suicidal ideation (relative risk 0.56) and one-quarter of the risk of suicide attempts 
(relative risk 0.24$).{ }^{7}$ Health care providers can support transgender patients with their legal sex marker change by providing letters of support, ${ }^{1}$ which may decrease the risk of suicidality. Both provincially and federally issued identification documents can be updated to reflect one's correct gender, including an " $X$ " or nonbinary gender option.

\section{Conclusion}

Transgender youth are overrepresented among the homeless youth population and experience high rates of mental health issues because of transphobia, structural violence and intersectional oppression. As seen in our case, health care providers can provide meaningful care for transgender youth with a history of trauma who are experiencing homelessness by addressing social determinants of health, transition-related goals, and any psychiatric and medical conditions concurrently. Because transgender youth live in all communities, it is important to ensure that those living in remote and rural Canada have the same access to appropriate and high-quality care as those living in large urban centres. ${ }^{7}$ Committing to this work, building longitudinal relationships that foster trust in the health care system, seeking transgenderinclusion training, and collaborating with an interdisciplinary team will allow health care providers to assist transgender youth in safely and successfully exiting homelessness.

\section{References}

1. Bourns A. Guidelines and protocols for hormone therapy and primary health care for trans clients. Rainbow Health Ontario. Toronto; 2015. Available: http:// sherbourne.on.ca/wp-content/uploads/2014/02/Guidelines-and-Protocols-for -Comprehensive-Primary-Care-for-Trans-Clients-2015.pdf (accessed 2019 June 10).

2. Winter S, Diamond M, Green J, et al. Transgender people: health at the margins of society. Lancet 2016;388:390-400.

3. Bauer GR, Travers R, Scanlon K, et al. High heterogeneity of HIV-related sexual risk among transgender people in Ontario, Canada: a province-wide respondent-driven sampling survey. BMC Public Health 2012;12:292-303.

4. Reisner SL, Poteat T, Keatley JA, et al. Global health burden and needs of transgender populations: a review. Lancet 2016;388:412-36.

5. Schuster MA, Reisner SL, Onorato SE. Beyond bathrooms - meeting the health needs of transgender people. N Engl J Med 2016;375:101-3.

6. Gaetz S, O'grady B, Kidd S, et al. Without a home: the national youth homelessness survey. Toronto: Canadian Observatory on Homelessness; 2016. Available: www.homelesshub.ca/YouthWithoutHome (accessed 2019 July 1).

7. Bauer GR, Scheim Al, Pyne J, et al. Intervenable factors associated with suicide risk in transgender persons: a respondent driven sampling study in Ontario, Canada. BMC Public Health 2015;15:525.

8. Rotondi NK, Bauer GR, Scanlon K, et al. Prevalence of and risk and protective factors for depression in female-to-male transgender Ontarians. Trans PULSE Project. Can J Commun Ment Health 2011;30:135-55.

9. Abramovich A. Preventing, reducing and ending LGBTQ2S youth homelessness: the need for targeted strategies. Soc Incl 2016;4:86-96.

10. De Cuypere G, Elaut E, Heylens G, et al. Long-term follow-up: psychosocial outcome of Belgian transsexuals after sex reassignment surgery. Sexologies 2006; 15:126-33.

\section{Competing interests: None declared.}

This article has been peer reviewed.

The authors have obtained patient consent.

Affiliations: Dalla Lana School of Public Health (Abramovich); Institute for Mental Health Policy Research (Abramovich), Centre for Addiction and Mental Health; Centre for Addiction and Mental Health (Lam); Department of Psychiatry (Lam), University of Toronto; Parkdale Queen West Community Health Centre (Chowdhury), Toronto, Ont.
Contributors: Alex Abramovich contributed to the overall conception and design of the manuscript. Muna Chowdhury contributed the specific details of the clinical case. Alex Abramovich drafted the manuscript, and all of the authors critically revised multiple versions before submission. All of the authors gave final approval of the version to be published and agreed to be accountable for all aspects of the work.

Acknowledgement: The authors thank Dr. Wayne Baici for reviewing the manuscript.

Correspondence to: Alex Abramovich, Alex.Abramovich@camh.ca 